\title{
Study of a CE Mechanism in Cyclic Chronopotentiometry with Spherical Electrodes
}

\author{
M. López-Tenés ", Á. Molina, J. M. Molina \\ Departamento de Química Física. Facultad de Química, Universidad de Murcia. \\ Espinardo, 30100. Murcia, Spain
}

Received 15 January 2003; accepted in revised form 21 February 2003

\begin{abstract}
The complete theory corresponding to a CE mechanism when applying cyclic chronopotentiometry to a spherical electrode of any size is developed. The influence of several variables on the transition time ratios, such as the electrode radius, rate constants of the homogeneous chemical reaction and current density, is discussed. A simple and practical criterion based on the variation of current density applied to the electrode is proposed for the detection of a CE mechanism.
\end{abstract}

Keywords: CE mechanism, cyclic chronopotentiometry, spherical electrodes, transition time ratios.

\section{Introduction}

The study of a CE mechanism, in which the charge transfer reaction follows a homogeneous chemical reaction, has been reported in the literature using several chronopotentiometric techniques such as constant current chronopotentiometry [1], chronopotentiometry with programmed current [2-4], and alternating current chronopotentiometry [5], and with planar and conventional sized spherical electrodes. In these techniques, only one current signal is applied to the electrode. However, electrochemical techniques in which the electrical perturbation (potential or current) is applied more than once are of great interest both analytically and kinetically [6,7]. Thus, a more exhaustive study of this type of

\footnotetext{
*Corresponding author. Fax: +3468364148; e-mail: manuela@um.es
} 
processes with kinetic complications can be made using cyclic chronopotentiometry, a classical electrochemical technique consisting of the application of several successive current steps of alternating signs to a determined electrode without the balance being recovered in the electrodesolution interphase. This technique was introduced by Herman and Bard in 1963 [8], who applied it to plane electrodes, and its use has been shown for the qualitative and quantitative study of electrode processes [9-13]. The technique was extended to conventional sized spherical electrodes in references [14-16]. In this paper, we have developed the theory concerning a $\mathrm{CE}$ mechanism in cyclic chronopotentiometry for spherical electrodes of any size, including planar electrodes and spherical ultramicroelectrodes as particular cases.

From this general theory, we analyse the response obtained in cyclic chronopotentiometry for a CE process and we discuss the influence of several variables, such as the electrode radius, on the transition time ratios $\left(\tau_{\text {step } j} / \tau_{\text {step } 1}\right)$, the evolution of which with the number of current steps applied can be used as a criterion to establish the presence of kinetic complications. We have proved that the characteristic behaviour of the transition time ratios corresponding to a $\mathrm{CE}$ process with the chemical rate constants can be also attained by changing the density current applied, something that is very easy to do experimentally. We also indicate how to obtain kinetic information of the process, and we conclude that cyclic chronopotentiometry has real advantages over other techniques for the study of a CE mechanism.

\section{Theory}

The reaction scheme for the CE mechanism can be written as (see notation)

$$
A \underset{k_{2}}{\stackrel{k_{1}}{\rightleftarrows}} O+n \mathrm{e}^{-} \underset{k_{b}}{\stackrel{k_{f}}{\rightleftarrows}} R
$$

We will consider a spherical static electrode of any size, and we will analyse the response of $\mathrm{CE}$ processes in cyclic chronopotentiometry. This technique consists of applying successive and alternating sign current steps in the following way 


$$
\left\{\begin{array}{cc}
I_{0}, & 0 \leq t_{1} \leq \tau_{1} \\
-I_{0}, & 0 \leq t_{2} \leq \tau_{2} \\
\vdots & \vdots \\
(-1)^{j+1} I_{0}, & 0 \leq t_{j} \leq \tau_{j} \\
\vdots & \vdots \\
(-1)^{k+1} I_{0}, & 0 \leq t_{k} \leq \tau_{k}
\end{array}\right.
$$

where, for the sake of simplicity, it has been supposed that all the current steps have the same absolute value, $I_{0}$. In this scheme, $t_{j}$ is the time during which a current step $j$ ( $j=1$ to $k$ ) is applied, and $\tau_{j}$ is the time for which the change in sign is produced, being the transition time corresponding to any reduction of species $O$ (forward transition times, $\tau_{1}, \tau_{3}, \tau_{5}, \ldots$ ) or to any oxidation of species $R$ (reverse transition times, $\tau_{2}, \tau_{4}, \tau_{6}, \ldots$ ). Thus, during the application of the $j$ th current step, the total time elapsed from the beginning of the experiment is given by

$$
t=\tau_{1}+\tau_{2}+\ldots+\tau_{j-1}+t_{j}
$$

Under these conditions, when the $j$ th current step is applied, the following equation system must be solved in order to obtain the expressions for the concentration profiles $\left(c_{i}^{j}\left(r, t_{j}\right), i=A, O, R\right)$ of species involved in a CE process

$$
\left\{\begin{array}{l}
\hat{\delta}_{A} c_{A}^{j}=-k_{1} c_{A}^{j}+k_{2} c_{O}^{j} \\
\hat{\delta}_{O} c_{O}^{j}=k_{1} c_{A}^{j}-k_{2} c_{O}^{j} \\
\hat{\delta}_{R} c_{R}^{j}=0
\end{array}\right.
$$

with $\hat{\delta}_{i}$ being the operator for the second Fick's law in spherical diffusion

$$
\hat{\delta}_{i}=\frac{\partial}{\partial t}-D_{i}\left(\frac{\partial^{2}}{\partial r^{2}}+\frac{2}{r} \frac{\partial}{\partial r}\right)
$$

where $D_{i}$ is the diffusion coefficient of species $i$, and $r$ is the distance from the centre of the electrode to any point in the solution. 
The boundary value problem is given by

$$
\begin{gathered}
\left.\begin{array}{l}
t_{j}=0, r \geq r_{0} \\
t_{j}>0, r \rightarrow \infty
\end{array}\right\} \begin{array}{c}
c_{A}^{j}\left(r, t_{j}\right)=c_{A}^{j-1}(r, t), c_{O}^{j}\left(r, t_{j}\right)=c_{O}^{j-1}(r, t), c_{R}^{j}\left(r, t_{j}\right)=c_{R}^{j-1}(r, t) \quad(j>1) \\
c_{A}^{1}\left(r, t_{1}\right)=c_{A}^{*}, c_{O}^{1}\left(r, t_{1}\right)=c_{O}^{*}, c_{R}^{1}\left(r, t_{1}\right)=c_{R}^{*}
\end{array} \\
t_{j}>0, r=r_{0}: \quad D_{O}\left(\frac{\partial c_{O}^{j}}{\partial r}\right)_{r=r_{0}}=-D_{R}\left(\frac{\partial c_{R}^{j}}{\partial r}\right)_{r=r_{0}}=\frac{(-1)^{j+1} I_{0}}{n F A} \\
D_{A}\left(\frac{\partial c_{A}^{j}}{\partial r}\right)_{r=r_{0}}=0
\end{gathered}
$$

where $c_{i}^{j-1}(r, t) \quad(i=A, O$ or $R)$ are the solutions for the $(j-1)$ th current step applied and $r_{0}$ is the electrode radius.

The solution of equation system (2) with the boundary value problem given by eqs. (4)-(6) has been carried out in the Appendix of this paper and the expressions for $c_{i}^{j}\left(r, t_{j}\right)$ have been obtained. From these, the equations for the special case $r=r_{0}$ (electrode surface) can be deduced. Thus, we find that the equations for the surface concentrations of species participating in the $\mathrm{CE}$ mechanism corresponding to the $j$ th ( $j=1$ to $k$ ) current step are

$$
\begin{aligned}
& \frac{c_{A}^{j}\left(r_{0}, t_{j}\right)}{c_{A}^{*}+c_{O}^{*}}=\frac{K_{A O}}{1+K_{A O}}\left\{1-N_{C E}\left\{\left(t_{1, j}\right)^{1 / 2}\left[S_{O}\left(\xi_{O}^{1, j}\right)-T_{O}\left(\xi_{O}^{1, j}, \chi^{1, j}\right)\right]+\right.\right. \\
& \left.\left.2 \sum_{n=2}^{j}(-1)^{n+1}\left(t_{n, j}\right)^{1 / 2}\left[S_{O}\left(\xi_{O}^{n, j}\right)-T_{O}\left(\xi_{o}^{n, j}, \chi^{n, j}\right)\right]\right\}\right\} \\
& \frac{c_{O}^{j}\left(r_{0}, t_{j}\right)}{c_{A}^{*}+c_{O}^{*}}=\frac{1}{1+K_{A O}}\left\{1-N_{C E}\left\{\left(t_{1, j}\right)^{1 / 2}\left[S_{O}\left(\xi_{O}^{1, j}\right)+K_{A O} T_{O}\left(\xi_{O}^{1, j}, \chi^{1, j}\right)\right]+\right.\right. \\
& \left.\left.2 \sum_{n=2}^{j}(-1)^{n+1}\left(t_{n, j}\right)^{1 / 2}\left[S_{O}\left(\xi_{O}^{n, j}\right)+K_{A O} T_{O}\left(\xi_{O}^{n, j}, \chi^{n, j}\right)\right]\right\}\right\} \\
& \frac{c_{R}^{j}\left(r_{0}, t_{j}\right)}{c_{A}^{*}+c_{O}^{*}}=\frac{c_{R}^{*}}{c_{A}^{*}+c_{O}^{*}}+\gamma N_{C E}\left\{\left(t_{1, j}\right)^{1 / 2} S_{R}\left(\xi_{R}^{1, j}\right)+2 \sum_{n=2}^{j}(-1)^{n+1}\left(t_{n, j}\right)^{1 / 2} S_{R}\left(\xi_{R}^{n, j}\right)\right\}
\end{aligned}
$$


where $c_{i}^{*}(i=A, O$ or $R)$ are the initial concentrations of species $i, K_{A O}$ is the equilibrium constant of the chemical reaction for the $\mathrm{CE}$ mechanism, which is given by

$$
K_{A O}=\frac{k_{2}}{k_{1}}=\frac{c_{A}^{*}}{c_{O}^{*}}
$$

and

$$
\begin{gathered}
N_{C E}=\frac{2 I_{0}}{n F A D_{O}^{1 / 2}\left(c_{A}^{*}+c_{O}^{*}\right)} \\
\gamma=\left(\frac{D_{O}}{D_{R}}\right)^{1 / 2} \\
t_{n, j}=\tau_{n}+\tau_{n+1}+\ldots+t_{j} \\
t_{j, j}=t_{j}
\end{gathered}
$$

The functions $S_{i}\left(\xi_{i}^{n, j}\right)$ and $T_{O}\left(\xi_{O}^{n, j}, \chi^{n, j}\right)(i=O$ or $R)$ are given by the following expressions

$$
\begin{gathered}
S_{i}\left(\xi_{i}^{n, j}\right)=\frac{1}{\xi_{i}^{n, j}}\left(1-\exp \left(\xi_{i}^{n, j} / 2\right)^{2} \operatorname{erfc}\left(\xi_{i}^{n, j} / 2\right)\right) \\
T_{O}\left(\xi_{O}^{n, j}, \chi^{n, j}\right)=\exp \left(-\chi_{O}^{n, j}\right) \frac{\xi_{O}^{n, j}}{4 \chi^{n, j}-\left(\xi_{O}^{n, j}\right)^{2}} \times \\
\left\{\exp \left(\xi_{O}^{n, j} / 2\right)^{2} \operatorname{erfc}\left(\xi_{O}^{n, j} / 2\right)-\exp \left(\chi^{n, j}\right)\left(1-\frac{2\left(\chi^{n, j}\right)^{1 / 2}}{\xi_{O}^{n, j}} \operatorname{erf}\left(\left(\chi^{n, j}\right)^{1 / 2}\right)\right)\right\}
\end{gathered}
$$

with variables $\xi_{i}^{n, j}$ and $\chi^{n, j}$ given by equations (A69) and (A70) in the Appendix by changing $t_{j}$ in these equations by $t_{n, j}$ (eq. (13)).

It is worthwhile highlighting that, due to the compact form of eqs. (15) and (16), the solutions presented in this paper are valid for spherical electrodes of any size, from spherical ultramicroelectrodes to planar electrodes. 
For planar electrodes $\left(r_{0} \rightarrow \infty\right.$, i.e. $\xi_{i}^{n, j} \rightarrow 0$ ), eqs. (15) and (16) become

$$
\begin{gathered}
t_{n, j}^{1 / 2} S_{i}\left(\xi_{i}^{n, j} \rightarrow 0\right)=\frac{t_{n, j}^{1 / 2}}{\pi^{1 / 2}} \\
t_{n, j}^{1 / 2} T_{O}\left(\xi_{O}^{n, j} \rightarrow 0, \chi^{n, j}\right)=\frac{t_{n, j}^{1 / 2} e r f\left(\left(\chi^{n, j}\right)^{1 / 2}\right)}{2\left(\chi^{n, j}\right)^{1 / 2}}
\end{gathered}
$$

and if $\chi^{n, j}>>1$, then eq. (18) can be written as

$$
t_{n, j}^{1 / 2} T_{O}\left(\xi_{O}^{n, j} \rightarrow 0, \chi^{n, j}>>1\right)=\frac{1}{2\left(k_{1}+k_{2}\right)^{1 / 2}}
$$

For conventional sized spherical electrodes, when $\chi^{n, j}>>1$, from eq. (16) we obtain

$$
t_{n, j}^{1 / 2} T_{O}\left(\xi_{O}^{n, j}, \chi^{n, j}>>1\right)=\frac{r_{0}^{2}\left(k_{1}+k_{2}\right)^{1 / 2}-D_{O}^{1 / 2} r_{0}}{2 r_{0}^{2}\left(k_{1}+k_{2}\right)-2 D_{O}}
$$

For ultramicroelectrodes ( $r_{0} \rightarrow 0$ and $\xi_{i}^{n, j}>>1$ ), eqs. (15) and (16) are simplified to

$$
t_{n, j}^{1 / 2} S_{i}\left(\xi_{i}^{n, j} \gg>1\right)=t_{n, j}^{1 / 2} T_{O}\left(\xi_{O}^{n, j}>>1, \chi^{n, j}\right)=\frac{r_{0}}{2 D_{i}^{1 / 2}}
$$

The potential-time response for the $j$ th $(j=1$ to $k$ ) current step can be deduced by substituting the expressions obtained for the surface concentrations of the oxidized and the reduced species, $c_{O}^{j}\left(r_{0}, t_{j}\right)$ and $c_{R}^{j}\left(r_{0}, t_{j}\right)$ (eqs. (8) and (9)), in the Butler-Volmer equation

$$
\frac{(-1)^{j+1} I_{0}}{n F A}=k_{f} c_{O}^{j}\left(r_{0}, t_{j}\right)-k_{b} c_{R}^{j}\left(r_{0}, t_{j}\right)
$$

Thus, we obtain the following expression 


$$
\begin{aligned}
& N_{C E} \frac{D_{O}^{1 / 2}}{2 k^{0}} e^{\alpha \eta \eta\left(t_{j}\right)}=(-1)^{j+1} \times \\
& \left\{\frac{1}{1+K_{A O}}\left[1-N_{C E}\left\{\left(t_{1, j}\right)^{1 / 2}\left[S_{O}\left(\xi_{O}^{1, j}\right)+K_{A O} T_{O}\left(\xi_{O}^{1, j}, \chi^{1, j}\right)\right]+2 \sum_{n=2}^{j}(-1)^{n+1}\left(t_{n, j}\right)^{1 / 2}\left[S_{O}\left(\xi_{O}^{n, j}\right)+K_{A O} T_{O}\left(\xi_{O}^{n, j}, \chi^{n, j}\right)\right]\right\}\right]\right. \\
& \left.e^{\eta\left(t_{j}\right)}\left[\frac{c_{R}^{*}}{c_{A}^{*}+c_{O}^{*}}+\gamma N_{C E}\left\{\left(t_{1, j}\right)^{1 / 2} S_{R}\left(\xi_{R}^{1, j}\right)+2 \sum_{n=2}^{j}(-1)^{n+1}\left(t_{n, j}\right)^{1 / 2} S_{R}\left(\xi_{R}^{n, j}\right)\right\}\right]\right\}
\end{aligned}
$$

with

$$
\eta\left(t_{j}\right)=\frac{n F}{R T}\left(E\left(t_{j}\right)-E^{0^{\prime}}\right)
$$

Eq. (23) can be simplified in two limit cases:

- When $k^{0^{\prime}} \rightarrow \infty$ (reversible charge transfer reaction) we obtain, for any current step applied, the following expression

$$
\eta\left(t_{j}\right)=\ln \frac{1-N_{C E}\left\{\left(t_{1, j}\right)^{1 / 2}\left[S_{O}\left(\xi_{O}^{1, j}\right)+K_{A O} T_{O}\left(\xi_{O}^{1, j}, \chi^{1, j}\right)\right]+2 \sum_{n=2}^{j}(-1)^{n+1}\left(t_{n, j}\right)^{1 / 2}\left[S_{O}\left(\xi_{O}^{n, j}\right)+K_{A O} T_{O}\left(\xi_{O}^{n, j}, \chi^{n, j}\right)\right]\right\}}{\left(1+K_{A O}\right)\left\{\frac{c_{R}^{*}}{c_{A}^{*}+c_{O}^{*}}+\gamma N_{C E}\left\{\left(t_{1, j}\right)^{1 / 2} S_{R}\left(\xi_{R}^{1, j}\right)+2 \sum_{n=2}^{j}(-1)^{n+1}\left(t_{n, j}\right)^{1 / 2} S_{R}\left(\xi_{R}^{n, j}\right)\right\}\right\}}
$$

- When $k^{0^{\prime}} \ll<1$ (totally irreversible charge transfer reaction), the response obtained depends on the sign of the current step applied:

a) If $j$ is odd (the sign of the current is positive and a reduction process takes place)

$\eta\left(t_{j}\right)=\frac{1}{\alpha} \ln \frac{1-N_{C E}\left\{\left(t_{1, j}\right)^{1 / 2}\left[S_{O}\left(\xi_{O}^{1, j}\right)+K_{A O} T_{O}\left(\xi_{O}^{1, j}, \chi^{1, j}\right)\right]+2 \sum_{n=2}^{j}(-1)^{n+1}\left(t_{n, j}\right)^{1 / 2}\left[S_{O}\left(\xi_{O}^{n, j}\right)+K_{A O} T_{O}\left(\xi_{O}^{n, j}, \chi^{n, j}\right)\right]\right\}}{\left(1+K_{A O}\right) N_{C E} \frac{D_{O}^{1 / 2}}{2 k^{0^{\prime}}}}$ 
b) If $\mathrm{j}$ is even (the sign of the current is negative and an oxidation process takes place)

$$
\eta\left(t_{j}\right)=\frac{1}{\alpha-1} \ln \frac{\frac{c_{R}^{*}}{c_{A}^{*}+c_{O}^{*}}+\gamma N_{C E}\left\{\left(t_{1, j}\right)^{1 / 2} S_{R}\left(\xi_{R}^{1, j}\right)+2 \sum_{n=2}^{j}(-1)^{n+1}\left(t_{n, j}\right)^{1 / 2} S_{R}\left(\xi_{R}^{n, j}\right)\right\}}{N_{C E} \frac{D_{O}^{1 / 2}}{2 k^{0^{j}}}}
$$

The expressions corresponding to the transition time of the $j$ th current step $(j>1)$ can be obtained by making $c_{o}^{j}\left(r_{0}, t_{j}\right)=0$ in eq. (8) if $j$ is odd and $c_{R}^{j}\left(r_{0}, t_{j}\right)=0$ in eq. (9) if $j$ is even. Thus, we find

$$
\begin{aligned}
& \tau_{\text {odd }}^{1 / 2}= \\
& \frac{1-N_{C E}\left\{\left(t_{1, j}\right)^{1 / 2}\left[S_{O}\left(\xi_{O}^{1, j}\right)+K_{A O} T_{O}\left(\xi_{O}^{1, j}, \chi^{1, j}\right)\right]+2 \sum_{n=2}^{j-1}(-1)^{n+1}\left(t_{n, j}\right)^{1 / 2}\left[S_{O}\left(\xi_{O}^{n, j}\right)+K_{A O} T_{O}\left(\xi_{O}^{n, j}, \chi^{n, j}\right)\right]\right\}}{2 N_{C E}\left[S_{O}\left(\xi_{O}^{j}\right)+K_{A O} T_{O}\left(\xi_{O}^{j}, \chi^{j}\right)\right]} \\
& \tau_{\text {even }}^{1 / 2}=\frac{\frac{c_{R}^{*}}{c_{A}^{*}+c_{O}^{*}}+\gamma N_{C E}\left\{\left(t_{1, j}\right)^{1 / 2} S_{R}\left(\xi_{R}^{1, j}\right)+2 \sum_{n=2}^{j-1}(-1)^{n+1}\left(t_{n, j}\right)^{1 / 2} S_{R}\left(\xi_{R}^{n, j}\right)\right\}}{2 \gamma N_{C E} S_{R}\left(\xi_{R}^{j}\right)}
\end{aligned}
$$

In the particular case $j=1$, from eq. (8) we obtain

$$
\tau_{1}^{1 / 2}=\frac{1}{N_{C E}\left[S_{O}\left(\xi_{O}^{1}\right)+K_{A O} T_{O}\left(\xi_{O}^{1}, \chi^{1}\right)\right]}
$$

The equations deduced for the surface concentrations, potential-time response and transition time of a $\mathrm{CE}$ mechanism become those corresponding to a simple charge transfer reaction (E mechanism) when $K_{A O}=0$. This behaviour also occurs, for $K_{A O} \neq 0$, when $\chi^{n, j}=0$ or $\chi^{n, j} \rightarrow \infty$ (see Results and discussion). In these cases, our equations coincide with those obtained in reference [14]. 


\section{Experimental}

In cyclic chronopotentiometry, successive current steps are applied according to scheme (II). Their sign is alternately changed at a time which may be less than the transition time corresponding to the $j$ th current step, $\tau_{j}$, or equal to it. We will consider that the current is reversed when the transition time $\tau_{j}$ is reached, which is actually the most common case in practice.

Fig. 1.a shows the variation of the surface concentration of species $O$ and $R$, participating in the electrochemical reaction. In a $\mathrm{CE}$ mechanism, $O$ is the oxidized species and its concentration becomes zero at the surface of the electrode at odd transition times $\left(\tau_{1}, \tau_{3}, \tau_{5}, \ldots\right)$, while $R$ is the reduced species and its concentration becomes zero at the surface of the electrode at even transition times $\left(\tau_{2}, \tau_{4}, \tau_{6}, \ldots\right)$.

Figs. 1.b and 1.c show the typical potential-time response for a CE mechanism in a cyclic chronopotentiometric experiment in the case of a reversible charge transfer reaction (Fig. 1.b) and in the contrary case of an irreversible electrochemical reaction (Fig. 1.c). In these figures, the values of the transition times can be also observed.

In cyclic chronopotentiometry, it is of great interest to study the variation of the transition time ratios, defined with respect to the transition time of the first electrochemical reaction, $\tau_{1}$, as

$$
a_{j}=\frac{\tau_{j}}{\tau_{1}}
$$

with the number of alternating current steps applied, $j$, since it allows us to characterize the electrode process. It is useful to plot the transition time ratios vs. $j$ for the CE mechanism, as well as those obtained for an E mechanism, which is taken as a reference. 


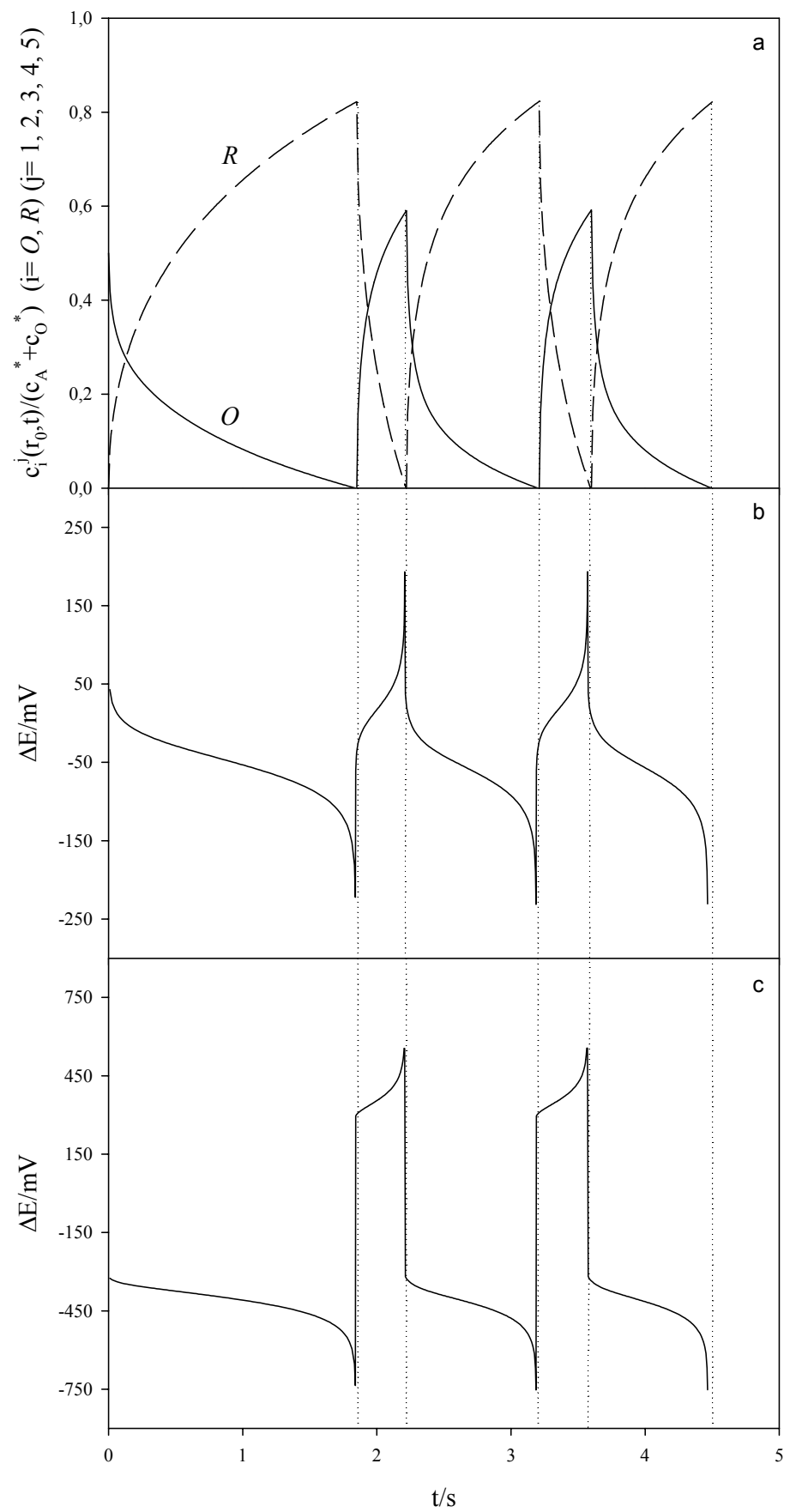

Figure 1.a. Variation of the surface concentration of species $O$ (solid line) and $R$ (dashed line) with time in cyclic chronopotentiometry $(j=1,2,3,4,5)$ for a $\mathrm{CE}$ mechanism (eqs. (8) and (9)). $K_{A O}=1, k_{1}+k_{2}=15 \mathrm{~s}^{-1}, N_{C E}=1.5 \mathrm{~s}^{-1 / 2}, D_{O}=10^{-5} \mathrm{~cm}^{2}$ $\mathrm{s}^{-1}, \gamma=1, c_{R}^{*}=0, r_{0}=10^{-2} \mathrm{~cm}$.

Figure 1.b. Potential-time curves corresponding to the application of five current steps for a CE mechanism with reversible charge transfer reaction (Eq. (25)). $T=298 \mathrm{~K}$, $n=1$. Other conditions as in Fig. 1.a.

Figure 1.c. Potential-time curves corresponding to the application of five current steps for a CE mechanism with irreversible charge transfer reaction (Eq. (26)). $k^{0^{\prime}}=10^{-5} \mathrm{~cm}$ $\mathrm{s}^{-1}, \alpha=0.5$. Other conditions as in Fig. 1.b. 
As can be deduced from eq. (28), the transition time obtained for a $\mathrm{CE}$ mechanism when a reduction process takes place ( $j$ odd) is given as a function of the equilibrium and rate constants of the chemical reaction. However, when an even current step is applied (eq. (29)), the transition time does not depend explicitly on these parameters. Thus, odd transition time ratios show a behaviour quite different to that observed for even ones, and that is why they need to be studied separately.

Fig. 2 shows the influence of $k_{1}+k_{2}$ on the variation of the transition time ratios with the number of current steps applied, considering a planar electrode and $K_{A O}=2$.

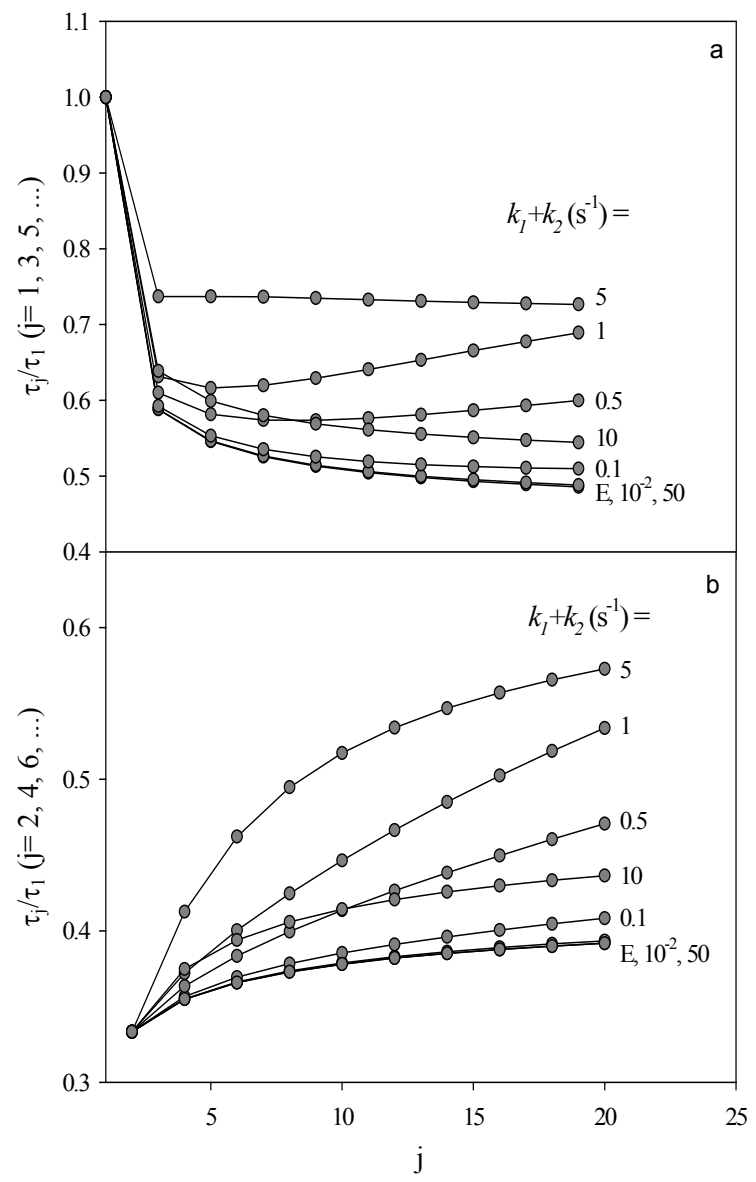

Figure 2. Influence of the rate constants on the variation of $\tau_{j} / \tau_{1}$ with $j$ for a $\mathrm{CE}$ mechanism in a planar electrode (Eqs. (28)-(30) and (17), (18)). $K_{A O}=2$. The values of $k_{1}+k_{2}\left(\right.$ in s $\left.^{-1}\right)$ are shown on the curves. The curve with label "E" corresponds to the behaviour of a simple E mechanism. a) $j$ odd; b) $j$ even. Other conditions as in Fig. 1.a. 
Fig. 2.a corresponds to odd values of $j$ and figure 2.b to $j$ even. Both figures show that relations $a_{j}$ are coincident with those obtained for an E mechanism (curves labelled with " $E$ " in figure) in two cases:

1. For $k_{1}+k_{2} \leq 10^{-2} \mathrm{~s}^{-1}$ (immobile chemical equilibrium), species $A$ and $O$ do not interconvert chemically. Consequently, $A$ merely acts as a chemically inert component of the system, and hence we observe a response which corresponds to an $\mathrm{E}$ mechanism with an initial concentration of species $O$ equal to $c_{O}^{*}$ (bulk concentration of species $O$ before the first current step is applied).

2. For $k_{1}+k_{2} \geq 50 \mathrm{~s}^{-1}$, the chemical equilibrium is totally mobile, and the system behaves as an $\mathrm{E}$ mechanism with an initial concentration of electroactive species $O$ equal to $c_{A}^{*}+c_{O}^{*}$. Thus, even though absolute transition times are greater than those in the situation above, the transition time ratios are identical in both cases.

For intermediate values of $k_{1}+k_{2}$, it can be observed in figures 2.a and 2.b that $a_{j}$ are greater than those corresponding to an $\mathrm{E}$ mechanism for any value of $j$. However, both figures show a different behaviour. Thus, for even values of $j$, the transition time ratios always increase with the number of current steps applied (Fig 2.b), while, if $j$ is odd (Fig. 2.a), the ratios $a_{j}$ exhibit the most characteristic behaviour of the CE mechanism: there is a range of values of $k_{1}+k_{2}\left(0 \leq k_{1}+k_{2} \leq\right.$ 5 in Fig. 2.a) for which the transition time ratios first decrease but then increase with the growing number of current steps applied. As can be observed, this does not occur with an E mechanism $\left(a_{j}\right.$ always decrease with $j$ ). Therefore, it is possible to characterize a $\mathrm{CE}$ mechanism by changing the rate constants, which can be done by modifying the experimental conditions (such as $\mathrm{pH}$ in the case of the reduction of an acid in a buffered solution or the concentration of ligand in the case of the reduction of a metal complex) if the chemical reaction is of pseudo-first order. 
The existence of a CE mechanism can be shown as well if we change the current density applied to the electrode, that is, if we change the variable $N_{C E}$ (eq. (11)). In Fig. 3 we have plotted the transition time ratios corresponding to a $\mathrm{CE}$ mechanism (planar electrode) vs. $j$ ( $j$ odd in Fig. 3.a and $j$ even in Fig. 3.b) for several values of $N_{C E}$. As in Fig. 2, the curve labelled with "E" corresponds to an E mechanism.

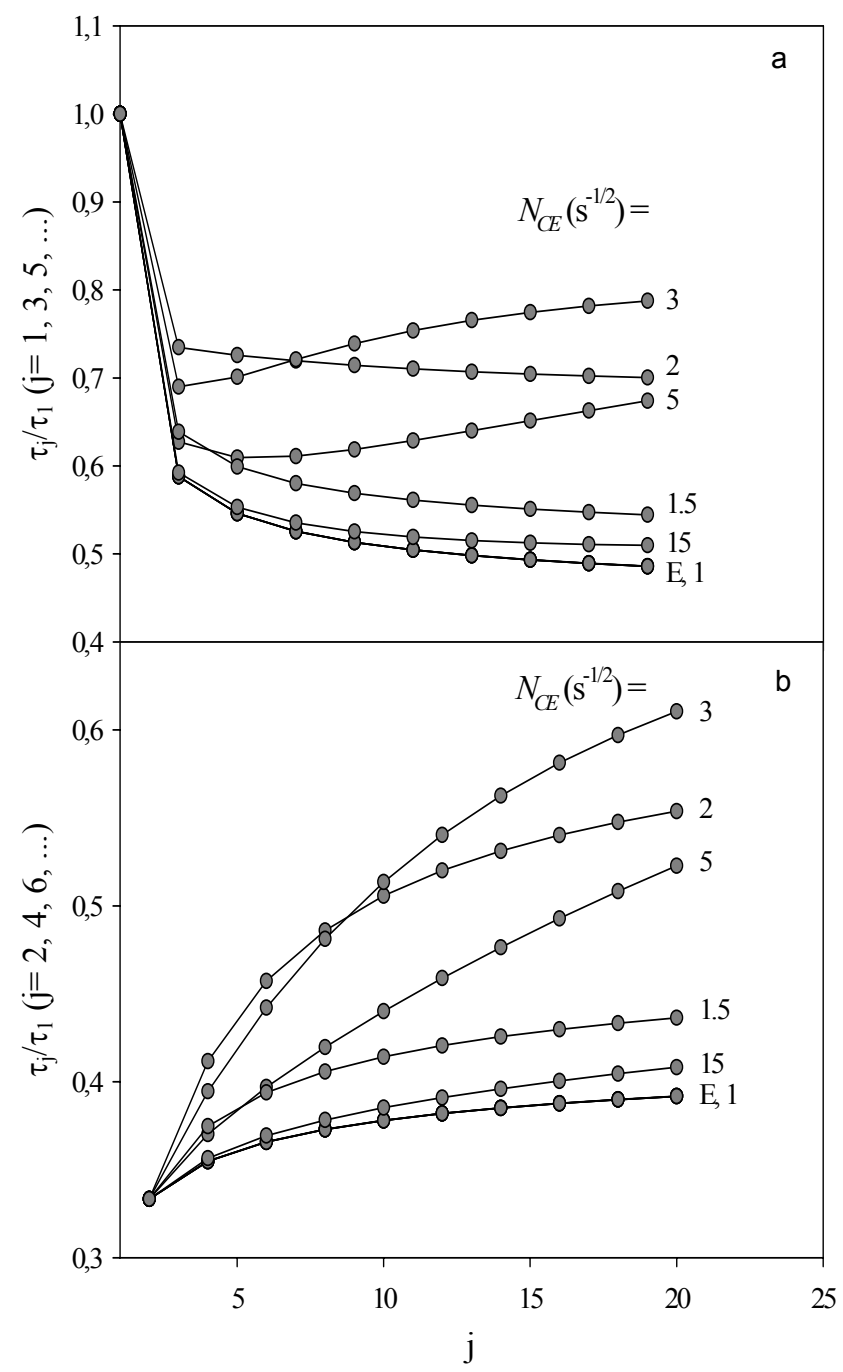

Figure 3. Influence of the current density on the variation of $\tau_{j} / \tau_{1}$ with $j$ for a $\mathrm{CE}$ mechanism in a planar electrode (eqs. (28)-(30) and (17), (18)). $k_{1}+k_{2}=10 \mathrm{~s}^{-1}$. The values of $N_{C E}\left(\right.$ in s$^{-1 / 2}$ ) are shown on the curves. a) $j$ odd; b) $j$ even. The curve with label "E" corresponds to the behaviour of a simple E mechanism. Other conditions as in Fig. 2. 
This figure has been prepared with a value of the rate constants $\left(k_{1}+k_{2}=10 \mathrm{~s}^{-1}\right)$ for which the odd transition time ratios do not show the typical behaviour of a CE mechanism in the conditions of Fig. 2.a. From the analysis of Fig. 3.a we can conclude that:

a) The variation of $N_{C E}$ has an influence on the ratios $a_{j}$. This does not occur in an E mechanism, where the transition time ratios are independent on the current density applied for planar electrodes (and a similar behaviour is observed for spherical electrodes).

b) The transition time ratios show the peculiar behaviour described above for high values of $N_{C E}$, and so we can characterize a CE mechanism by changing the current density applied, what is very easy to do experimentally.

Thus, an increase of $N_{C E}$ achieves the same effect as a possible or hypothetic diminution of $k_{1}+k_{2}$, and therefore, it is a way of externally modifying, through an experimental variable, the mobility of the chemical equilibrium coupled to the charge transfer reaction.

In order to analyse the influence of the sphericity, in Fig. 4 we have plotted the variation of the transition time ratios corresponding to a $\mathrm{CE}$ mechanism with $j$, for several values of $r_{0}$.

As can be observed, the ratios $a_{j}$ decrease always when $r_{0}$ diminishes. The behaviour is qualitatively the same as that shown for a planar electrode, but, from a quantitative point of view, the effect exerted by the electrode radius on the transition time ratios is very important, and becomes greater with the growing number of current steps applied. Therefore, it is specially important in cyclic chronopotentiometry, and it is consequently of great interest to have deduced equations available that take into account the sphericity of the electrode. 


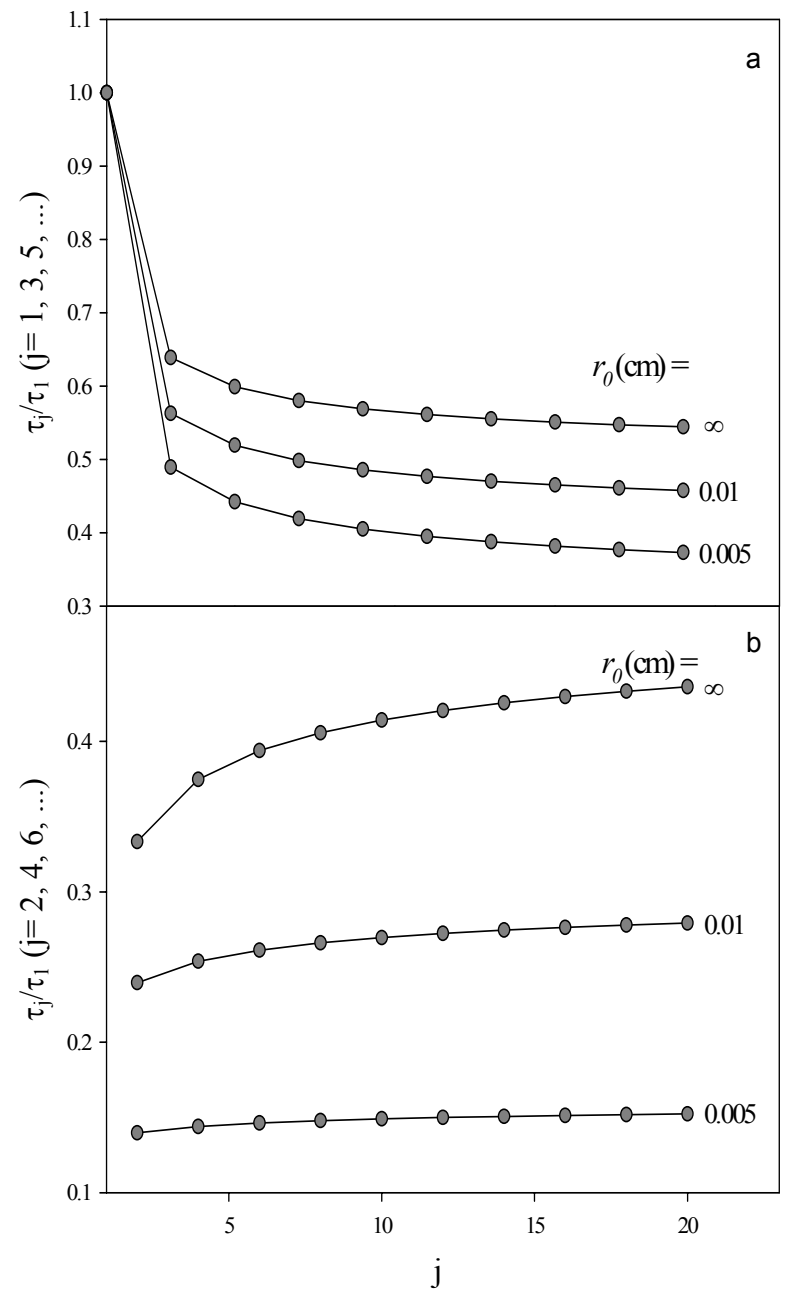

Figure 4. Influence of the sphericity on the variation of $\tau_{j} / \tau_{1}$ with $j$ for a $\mathrm{CE}$ mechanism (Eqs. (28)-(30)). $k_{1}+k_{2}=10 \mathrm{~s}^{-1}$. The values of $r_{0}$ (in $\mathrm{cm}$ ) are shown on the curves. The curve with $r_{0} \rightarrow \infty$ corresponds to a planar electrode. a) $j$ odd; b) $j$ even. Other conditions as in Fig. 2.

The study of the transition time ratios can also be used to calculate the rate constants of the chemical reaction coupled to the charge transfer reaction, as has been pointed out in reference [9]. The rate constants can be determined by comparing theoretical and experimental $\tau_{j} / \tau_{1}$ vs. $j$ curves. This method is more advantageous than that described in references $[3,4,16]$, where constant current and current reversal chronopotentiometry are used, because in the first case we obtain all the kinetic information from only one transition time measurement $\left(\tau_{1}\right)$, and in the case of current reversal, the ratio $\tau_{2} / \tau_{1}$ is independent on kinetic 
parameters. However, in cyclic chronopotentiometry we can apply as many current steps as we want to.

\section{Acknowledgements}

The authors greatly appreciate the financial support provided by the Dirección General Científica y Técnica (Project No. BQU2000-0231), and by the Fundación Séneca (Projects 00696/CV/99 and AR 28-02698/FS/02). Also J. M. Molina thanks Fundación Séneca for the grant received.

\section{Notation and definitions}

$k_{f}, k_{b}$ heterogeneous rate constants of forward (reduction) and backward (oxidation) charge transfer processes

$k^{0^{\prime}} \quad$ apparent heterogeneous rate constant of charge transfer at $E^{0^{\prime}}$

$\alpha \quad$ charge transfer coefficient

$k_{1}, k_{2}$ rate constants of the homogeneous chemical reaction

$K_{A O} \quad$ equilibrium constant of the chemical reaction $\left(=k_{2} / k_{1}\right)$

$r$ distance from the centre of the spherical electrode to any point in the solution

$r_{0} \quad$ electrode radius of the spherical electrode

$c_{i}^{j}(r, t)$ concentration profile of species $i(i=A, O$ or $R)$ when a $j$ current step is applied

$c_{i}^{j}\left(r_{0}, t\right)$ surface concentration of species $i(i=A, O$ or $R)$ when a $j$ current step is applied

$c_{i}^{*} \quad$ bulk concentration of species $i(i=A, O$, or $R)$

$t \quad$ time elapsed between application of the first and the $j$ th current step $\left(=\tau_{1}+\tau_{2}+\ldots+t_{j}\right)$

$t_{n, j} \quad$ time elapsed between application of the $n$th and the $j$ th current step $\left(=\tau_{n}+\tau_{n+1}+\ldots+t_{j}\right)$

$t_{j} \quad$ time during which a $j$ current step is applied $\left(0 \leq t_{j} \leq \tau_{j}\right)$

$a_{j} \quad$ transition time ratios

$\tau_{j} \quad$ transition time of the $j$ th current step

$n \quad$ number of the electrons transferred in the electrochemical reaction

$F \quad$ Faraday constant

$A \quad$ area of the electrode

$I_{0} \quad$ absolute value of the current step applied

$D_{i} \quad$ diffusion coefficient of species $i\left(i=A, O\right.$ or $R$ and $\left.D_{A}=D_{O}\right)$

$N_{C E} \quad=2 I_{0} / n F A D_{O}^{1 / 2}\left(c_{A}^{*}+c_{O}^{*}\right)$ 


$$
\begin{array}{lll}
\gamma & =\left(D_{O} / D_{R}\right)^{1 / 2} \\
\xi_{i}^{n, j} & \text { dimensionless parameter of spherical diffusion }\left(=2 \sqrt{D_{i} t_{n, j}} / r_{0}\right) \\
\chi^{n, j} & \text { dimensionless parameter referring to the chemical reaction } \\
& \left(=\left(k_{1}+k_{2}\right) t_{n, j}\right) \\
E\left(t_{j}\right) & \text { time-dependent potential } \\
E^{0^{\prime}} & \text { formal potential of the electrode reaction } \\
\Delta E & =E\left(t_{j}\right)-E^{0^{\prime}}
\end{array}
$$

\section{References}

1. P. Delahay, T. Berzins, J. Am. Chem. Soc. 75 (1953) 2486-2493.

2. R. Kant, S.K. Rangarajan, J. Electroanal. Chem. 265 (1989) 39-65.

3. A. Molina, M. López-Tenés, Collect. Czech. Chem. Comm. 56 (1991) 1-19.

4. A. Molina, M. López-Tenés, C. Serna, J. Electroanal. Chem. 346 (1993) 5371.

5. M.L. Alcaraz, A. Molina, M. López-Tenés, Electrochim. Acta 42 (1997) 1351-1359.

6. D.D. McDonald, Transient Techniques in Electrochemistry, Plenum Press, New York, 1977, chapter 3.

7. A.J. Bard, L.R. Faulkner, Electrochemical Methods, Wiley, New York, 2001, chapter 5 .

8. H.B. Herman, A.J. Bard, Anal. Chem. 35 (1963) 1121-1125.

9. H.B. Herman, A.J. Bard, Anal. Chem. 36 (1964) 510-514; 36 (1964) 971-975.

10. H.B. Herman, A.J. Bard, J. Electrochem. Soc. 115 (1968) 1028-1033.

11.A. Molina, M.L. Alcaraz, F. Saavedra, J. González, Electrochim. Acta 44 (1998) 1263-1272.

12.L.M. Abrantes, J. González, A. Molina, F. Saavedra, Electrochim. Acta 45 (1999) 457-468.

13. A. Molina, J. González, F. Saavedra, L.M. Abrantes, Electrochim. Acta 45 (1999) 761-773.

14. A. Molina, J. González, C. Serna, L. Camacho, J. Math. Chem. 20 (1996) 168-181. 
15. J. González, A. Molina, F. Martínez-Ortiz, C. Serna, J. Electroanal. Chem. 440 (1997) 111-123.

16. A. Molina, J. González, M. López-Tenés, J. Math. Chem. 23 (1998) 277-296.

17. J. Koutecký, Czech. J. Phys. 2 (1953) 50-55.

18. A. Molina, C. Serna, L. Camacho, J. Electroanal. Chem. 394 (1995) 1-6.

\section{Application of the first current step}

\section{Appendix}

When a spherical electrode of any size is considered, the mass transport to the electrode surface when the first current step is applied is described by the differential equation system (eq. (2) with $j=1$ )

$$
\left\{\begin{array}{l}
\hat{\delta}_{A} c_{A}^{1}=-k_{1} c_{A}^{1}+k_{2} c_{O}^{1} \\
\hat{\delta}_{O} c_{O}^{1}=k_{1} c_{A}^{1}-k_{2} c_{O}^{1} \\
\hat{\delta}_{R} c_{R}^{1}=0
\end{array}\right.
$$

where $\hat{\delta}_{i}(i=A, O$ or $R)$ is the operator for the second Fick's law given by eq. (3).

The boundary value problem (Eqs. (4)-(6)) is given by:

$$
\begin{gathered}
\left.\begin{array}{c}
t_{1}=0, r \geq r_{0} \\
t_{1}>0, r \rightarrow \infty
\end{array}\right\} \quad c_{A}^{1}=c_{A}^{*}, \quad c_{O}^{1}=c_{O}^{*}, \quad c_{R}^{1}=c_{R}^{*} \\
t_{1}>0, r=r_{0}: \quad D_{O}\left(\frac{\partial c_{O}^{1}}{\partial r}\right)_{r=r_{0}}=-D_{R}\left(\frac{\partial c_{R}^{1}}{\partial r}\right)_{r=r_{0}}=\frac{I_{0}}{n F A} \\
D_{A}\left(\frac{\partial c_{A}^{1}}{\partial r}\right)_{r=r_{0}}=0
\end{gathered}
$$

By introducing the variables:

$$
\begin{gathered}
\zeta^{1}\left(r, t_{1}\right)=c_{A}^{1}\left(r, t_{1}\right)+c_{O}^{1}\left(r, t_{1}\right) \\
\phi^{1}\left(r, t_{1}\right)=\left(c_{A}^{1}\left(r, t_{1}\right)-K_{A O} c_{O}^{1}\left(r, t_{1}\right)\right) e^{\left(k_{1}+k_{2}\right) t_{1}}
\end{gathered}
$$

and with the assumption

$$
D_{A}=D_{O} \neq D_{R}
$$


the differential equation system (A1) and the boundary value problem (eqs. (A2)(A4)) are transformed into:

$$
\begin{gathered}
\hat{\delta}_{O} \zeta^{1}=\hat{\delta}_{O} \phi^{1}=\hat{\delta}_{R} c_{R}^{1}=0 \\
\left.\begin{array}{c}
t_{1}=0, r \geq r_{0} \\
t_{1}>0, r \rightarrow \infty
\end{array}\right\} \quad \zeta^{1}=c_{A}^{*}+c_{O}^{*}, \quad \phi^{1}=0, \quad c_{R}^{1}=c_{R}^{*} \\
t_{1}>0, r=r_{0}: \quad D_{O}\left(\frac{\partial \zeta^{1}}{\partial r}\right)_{r=r_{0}}=-D_{R}\left(\frac{\partial c_{R}^{1}}{\partial r}\right)_{r=r_{0}}=\frac{I_{0}}{n F A} \\
\left(\frac{\partial \phi^{1}}{\partial r}\right)_{r=r_{0}}=-K_{A O} e^{\left(k_{1}+k_{2}\right) t_{1}} \frac{I_{0}}{n F A D_{O}}
\end{gathered}
$$

This problem can also be solved by introducing the variables

$$
\begin{aligned}
& u^{1}=\frac{\zeta^{1}}{c_{A}^{*}+c_{O}^{*}} \frac{r}{r_{0}} \\
& v^{1}=\frac{\phi^{1}}{c_{A}^{*}+c_{O}^{*}} \frac{r}{r_{0}}
\end{aligned}
$$

In such a way, the differential equation system (A8) becomes

$$
\hat{\delta}_{O} u^{1}=\hat{\delta}_{O} v^{1}=\hat{\delta}_{R} c_{R}^{1}=0
$$

where $\hat{\delta}_{i}$ is now

$$
\hat{\delta}_{i}=\frac{\partial}{\partial t}-D_{i} \frac{\partial^{2}}{\partial r^{2}}
$$

By supposing that $\zeta^{1}, \phi^{1}$, and $c_{R}^{1}$ have the form:

$$
\begin{gathered}
\zeta^{1}\left(r, t_{1}\right)=\left(c_{A}^{*}+c_{O}^{*}\right)+\sum_{p, q=0} \rho_{p, q}^{1}\left(s_{O}^{1}\right)\left(\xi_{O}^{1}\right)^{p}\left(\chi^{1}\right)^{q / 2} \\
\phi^{1}\left(r, t_{1}\right)=\sum_{p, q=0} \delta_{p, q}^{1}\left(s_{O}^{1}\right)\left(\xi_{O}^{1}\right)^{p}\left(\chi^{1}\right)^{q+1 / 2} \\
c_{R}^{1}\left(r, t_{1}\right)=c_{R}^{*}+\sum_{p, q=0} \sigma_{p, q}^{1}\left(s_{R}^{1}\right)\left(\xi_{R}^{1}\right)^{p}\left(\chi^{1}\right)^{q / 2}
\end{gathered}
$$


where

$$
\begin{gathered}
s_{i}^{1}=\frac{r-r_{0}}{2 \sqrt{D_{i} t_{1}}} \\
\xi_{i}^{1}=\frac{2 \sqrt{D_{i} t_{1}}}{r_{0}} \\
\chi^{1}=\left(k_{1}+k_{2}\right) t_{1}
\end{gathered}
$$

and using the dimensionless parameters method [17] to solve the differential equation system (A8), we obtain the following solutions:

$$
\begin{aligned}
& \rho_{p, q}^{1}\left(s_{O}^{1}\right)=0 \quad \text { unless } \quad q=1 \\
& \rho_{0,1}^{1}\left(s_{O}^{1}\right)=-\frac{N_{C E}\left(c_{A}^{*}+c_{O}^{*}\right)}{\left(k_{1}+k_{2}\right)^{1 / 2}}\left\{\frac{\Psi_{1}\left(s_{O}^{1}\right)}{p_{1}}\right\} \\
& \rho^{1}{ }_{1,1}\left(s_{O}^{1}\right)=-\frac{N_{C E}\left(c_{A}^{*}+c_{O}^{*}\right)}{\left(k_{1}+k_{2}\right)^{1 / 2}}\left\{\frac{\Psi_{2}\left(s_{O}^{1}\right)}{2}-\Psi_{0}\left(s_{O}^{1}\right)\right\} \\
& \rho_{2,1}^{1}\left(s_{O}^{1}\right)=-\frac{N_{C E}\left(c_{A}^{*}+c_{O}^{*}\right)}{\left(k_{1}+k_{2}\right)^{1 / 2}}\left\{\frac{\Psi_{3}\left(s_{O}^{1}\right)}{p_{3}}-\frac{p_{0} \Psi_{1}\left(s_{O}^{1}\right)}{2}+\frac{p_{0} \Psi_{-1}\left(s_{O}^{1}\right)}{4}\right\} \\
& \rho^{1}{ }_{3,1}\left(s_{O}^{1}\right)=-\frac{N_{C E}\left(c_{A}^{*}+c_{O}^{*}\right)}{\left(k_{1}+k_{2}\right)^{1 / 2}}\left\{\frac{15}{32} \Psi_{4}\left(s_{O}^{1}\right)-\Psi_{2}\left(s_{O}^{1}\right)+\frac{\Psi_{0}\left(s_{O}^{1}\right)}{2}\right\} \\
& \text { ! } \\
& \delta_{0, q}^{1}\left(s_{O}^{1}\right)=\frac{K_{A O} N_{C E}\left(c_{A}^{*}+c_{O}^{*}\right)}{q !\left(k_{1}+k_{2}\right)^{1 / 2}} \frac{\Psi_{2 q+1}\left(s_{O}^{1}\right)}{p_{2 q+1}} \\
& \delta_{1, q}^{1}\left(s_{O}^{1}\right)=\frac{K_{A O} N_{C E}\left(c_{A}^{*}+c_{O}^{*}\right)}{2 q !\left(k_{1}+k_{2}\right)^{1 / 2}}\left\{\frac{2 q+1}{2 q+2} \Psi_{2 q+2}\left(s_{O}^{1}\right)-\Psi_{2 q}\left(s_{O}^{1}\right)\right\} \\
& \delta_{2, q}^{1}\left(s_{O}^{1}\right)=\frac{K_{A O} N_{C E}\left(c_{A}^{*}+c_{O}^{*}\right)}{4 q !\left(k_{1}+k_{2}\right)^{1 / 2}}\left\{\frac{4(q+1)}{p_{2 q+3}} \Psi_{2 q+3}\left(s_{O}^{1}\right)-2 p_{2 q} \Psi_{2 q+1}\left(s_{O}^{1}\right)+p_{2 q} \Psi_{2 q-1}\left(s_{O}^{1}\right)\right\}
\end{aligned}
$$




$$
\begin{aligned}
S_{3, q}^{1}\left(s_{O}^{1}\right) & =\frac{K_{A O} N_{C E}\left(c_{A}^{*}+c_{O}^{*}\right)}{4 q !\left(k_{1}+k_{2}\right)^{1 / 2}} \times \\
& \left\{\frac{8 q^{3}-32 q^{2}+40 q+15}{(2 q+4)(2 q+2)} \Psi_{2 q+4}\left(s_{O}^{1}\right)-(6 q+4) \Psi_{2 q+2}\left(s_{O}^{1}\right)+(6 q+2) \Psi_{2 q}\left(s_{O}^{1}\right)-2 q \Psi_{2 q-2}\left(s_{O}^{1}\right)\right\}
\end{aligned}
$$

$$
\sigma_{p, 1}^{1}\left(s_{R}^{1}\right)=-\gamma \rho_{p, 1}^{1}\left(s_{R}^{1}\right)
$$

where $\gamma$ is given by eq. (12) and functions $\rho_{p, 1}^{1}\left(s_{R}^{1}\right)$ are given by eqs. (A22)(A26) by changing $s_{O}^{1}$ by $s_{R}^{1}$ (eq. (A19)). $\Psi\left(s_{i}^{1}\right) \quad(\mathrm{i}=\mathrm{O}, \mathrm{R})$ are the Koutecký functions and $p_{m}=\Gamma(1+m / 2) / \Gamma((1+m) / 2)$.

\section{Application of the second current step}

When the second current step $-I_{0}$ is applied (eqs. (2)-(6) with $j=2$ ), as this problem is linear, we assume that the expressions of the concentration profiles can be written:

$$
\begin{aligned}
& c_{A}^{2}\left(r, t_{2}\right)=c_{A}^{1}(r, t)+\tilde{c}_{A}^{2}\left(r, t_{2}\right) \\
& c_{O}^{2}\left(r, t_{2}\right)=c_{O}^{1}(r, t)+\tilde{c}_{O}^{2}\left(r, t_{2}\right) \\
& c_{R}^{2}\left(r, t_{2}\right)=c_{R}^{1}(r, t)+\tilde{c}_{R}^{2}\left(r, t_{2}\right)
\end{aligned}
$$

where (see eq. (1))

$$
t=\tau_{1}+t_{2}
$$

$c_{i}^{1}(r, t)(i=A, O$ or $R)$ are the solutions obtained for the application of the first current step, and $\tilde{c}_{i}^{2}\left(r, t_{2}\right)$ are the new unknown functions.

Therefore, if we define the new variables

$$
\begin{gathered}
\zeta^{2}=c_{A}^{2}+c_{O}^{2}=\zeta^{1}+\tilde{\zeta}^{2} \\
\phi^{2}=\left(c_{A}^{2}-K_{A O} c_{O}^{2}\right) e^{\left(k_{1}+k_{2}\right)\left(\tau_{1}+t_{2}\right)}=\phi^{1}+\tilde{\phi}^{2}
\end{gathered}
$$


where $\zeta^{1}$ and $\phi^{1}$ have already been obtained for the previous step, the boundary value problem has now the following, simplified form in terms only of the new unknown functions $\tilde{\zeta}^{2}$ and $\tilde{\phi}^{2}$ :

$$
\begin{gathered}
\left.\begin{array}{c}
t_{2}=0, r \geq r_{0} \\
t_{2}>0, r=r_{0}
\end{array}\right\} \quad D_{O}\left(\frac{\partial \tilde{\zeta}^{2}}{\partial r}\right)_{r=r_{0}}=-D_{R}\left(\frac{\partial \tilde{c}_{R}^{2}}{\partial r}\right)_{r=r_{0}}=-\frac{2 I_{0}}{n F A} \\
t_{2}>0, r=r_{0}: \quad \tilde{\phi}^{2}=\tilde{c}_{R}^{2}=0 \\
\left(\frac{\partial \tilde{\phi}^{2}}{\partial r}\right)_{r=r_{0}}=K_{A O} e^{\left(k_{1}+k_{2}\right)\left(\tau_{1}+t_{2}\right)} \frac{2 I_{0}}{n F A D_{O}}
\end{gathered}
$$

As can be observed, the boundary value problem which $\zeta^{1}$ must fulfil in the first current step (eq. (A10)) is similar to that corresponding to $\tilde{\zeta}^{2}$ in this second current step (eq. (A39)), changing $I_{0}$ by $-2 I_{0}$, while the boundary value problem that $\phi^{1}$ must fulfil in the first current step (eq. (A11)) is analogous to that fulfilled by $\tilde{\phi}^{2}$ in the second current step (eq. (A40)), except for the value of the constant $-2 e^{\left(k_{1}+k_{2}\right) \tau_{1}}$.

By supposing that

$$
\begin{aligned}
& \tilde{\zeta}^{2}\left(r, t_{2}\right)=\sum_{p, q=0} \rho_{p, q}^{2}\left(s_{O}^{2}\right)\left(\xi_{O}^{2}\right)^{p}\left(\chi^{2}\right)^{q / 2} \\
& \tilde{\phi}^{2}\left(r, t_{2}\right)=\sum_{p, q=0} \delta_{p, q}^{2}\left(s_{O}^{2}\right)\left(\xi_{O}^{2}\right)^{p}\left(\chi^{2}\right)^{q+1 / 2} \\
& \tilde{c}_{R}^{2}\left(r, t_{2}\right)=\sum_{p, q=0} \sigma_{p, q}^{2}\left(s_{R}^{2}\right)\left(\xi_{R}^{2}\right)^{p}\left(\chi^{2}\right)^{q / 2}
\end{aligned}
$$

and proceeding as in the previous current step, we find that

$$
\begin{gathered}
\rho_{p, q}^{2}\left(s_{O}^{2}\right)=-2 \rho_{p, q}^{1}\left(s_{O}^{2}\right) \\
\delta_{p, q}^{2}\left(s_{O}^{2}\right)=-2 e^{\left(k_{1}+k_{2}\right) \tau_{1}} \delta_{p, q}^{1}\left(s_{O}^{2}\right) \\
\sigma_{p, q}^{2}\left(s_{R}^{2}\right)=-\gamma \rho_{p, q}^{2}\left(s_{R}^{2}\right)
\end{gathered}
$$

where 


$$
\begin{array}{cc}
s_{i}^{2}=\frac{r-r_{0}}{2 \sqrt{D_{i} t_{2}}} \quad(i=O, R) \\
\xi_{i}^{2}=\frac{2 \sqrt{D_{i} t_{2}}}{r_{0}} \quad(i=O, R) \\
\chi^{2}=\left(k_{1}+k_{2}\right) t_{2}
\end{array}
$$

and functions $\rho_{p, q}^{1}\left(s_{O}^{2}\right)$ and $\delta_{p, q}^{1}\left(s_{O}^{2}\right)$ have the form given by equations (A22)(A30), by substituting $s_{O}^{1}$ by $s_{O}^{2}$.

The mathematical treatment used for the first and the second current steps can be easily generalised by induction for any number of current steps. Thus, for the $j$ th current step $(j>1)$, by applying the superposition principle $[14,16,18]$ we can express the solution for the differential equation system (2) in the form

$$
\begin{aligned}
& c_{A}^{j}\left(r, t_{j}\right)=c_{A}^{j-1}(r, t)+\tilde{c}_{A}^{j}\left(r, t_{j}\right) \\
& c_{O}^{j}\left(r, t_{j}\right)=c_{O}^{j-1}(r, t)+\tilde{c}_{O}^{j}\left(r, t_{j}\right) \\
& c_{R}^{j}\left(r, t_{j}\right)=c_{R}^{j-1}(r, t)+\tilde{c}_{R}^{j}\left(r, t_{j}\right)
\end{aligned}
$$

where

$$
c_{i}^{j-1}(r, t)=c_{i}^{1}(r, t)+\sum_{m=2}^{j-1} \tilde{c}_{i}^{m}\left(r, t_{m}\right) \quad(i=A, O, R)
$$

Thus, if we define the functions

$$
\begin{gathered}
\zeta^{j}=c_{A}^{j}+c_{O}^{j}=\zeta^{j-1}+\tilde{\zeta}^{j} \\
\phi^{j}=\left(c_{A}^{j}-K_{A O} c_{O}^{j}\right) e^{\left(k_{1}+k_{2}\right) t}=\phi^{j-1}+\tilde{\phi}^{j}
\end{gathered}
$$

with

$$
\begin{aligned}
& \zeta^{j-1}(r, t)=\zeta^{1}(r, t)+\sum_{m=2}^{j-1} \tilde{\zeta}^{m}\left(r, t_{m}\right) \\
& \phi^{j-1}(r, t)=\phi^{1}(r, t)+\sum_{m=2}^{j-1} \tilde{\phi}^{m}\left(r, t_{m}\right)
\end{aligned}
$$

where $t$ is given by (eq (1)) 


$$
t=\tau_{1}+\tau_{2}+\ldots+t_{j}
$$

from eqs. (A36)-(A40) it can be demonstrated that the boundary value problem has the generalised form:

$$
\begin{gathered}
\left.\begin{array}{c}
t_{j}=0, r \geq r_{0} \\
t_{j}>0, r=r_{0}
\end{array}\right\} \quad \tilde{\zeta}^{j}=\tilde{\phi}^{j}=\tilde{c}_{R}^{j}=0 \\
t_{j}>0, r=r_{0}: \quad D_{O}\left(\frac{\partial \tilde{\zeta}^{j}}{\partial r}\right)_{r=r_{0}}=-D_{R}\left(\frac{\partial \tilde{c}_{R}^{j}}{\partial r}\right)_{r=r_{0}}=(-1)^{j+1} \frac{2 I_{0}}{n F A} \\
\left(\frac{\partial \tilde{\phi}^{j}}{\partial r}\right)_{r=r_{0}}=(-1)^{j} K_{A O} e^{\left(k_{1}+k_{2}\right) t} \frac{2 I_{0}}{n F A D_{O}}
\end{gathered}
$$

As can be deduced from eqs. (A38)-(A40) and (A59)-(A61), the partial solutions $\widetilde{\zeta}^{j}\left(r, t_{j}\right), \tilde{\phi}^{j}\left(r, t_{j}\right)$ and $\tilde{c}_{R}^{j}\left(r, t_{j}\right)$ are formally identical to $\widetilde{\zeta}^{2}\left(r, t_{2}\right), \tilde{\phi}^{2}\left(r, t_{2}\right)$ and $\tilde{c}_{R}^{j}\left(r, t_{2}\right)$, i.e. the superposition principle is fulfilled. Therefore, if we suppose that

$$
\begin{aligned}
& \tilde{\zeta}^{j}\left(r, t_{j}\right)=\sum_{p, q=0} \rho_{p, q}^{j}\left(s_{O}^{j}\right)\left(\xi_{O}^{j}\right)^{p}\left(\chi^{j}\right)^{q / 2} \\
& \tilde{\phi}^{j}\left(r, t_{j}\right)=\sum_{p, q=0} \delta_{p, q}^{j}\left(s_{O}^{j}\right)\left(\xi_{O}^{j}\right)^{p}\left(\chi^{j}\right)^{q+1 / 2} \\
& \tilde{c}_{R}^{j}\left(r, t_{j}\right)=\sum_{p, q=0} \sigma_{p, q}^{j}\left(s_{R}^{j}\right)\left(\xi_{R}^{j}\right)^{p}\left(\chi^{j}\right)^{q / 2}
\end{aligned}
$$

it is clear that we can express the solutions in the general form

$$
\begin{gathered}
\rho_{p, q}^{j}\left(s_{O}^{j}\right)=2(-1)^{j+1} \rho_{p, q}^{1}\left(s_{O}^{j}\right) \\
\delta_{p, q}^{j}\left(s_{O}^{j}\right)=2(-1)^{j+1} e^{\left(k_{1}+k_{2}\right) \sum_{l=1}^{j-1} \tau_{1}} \delta_{p, q}^{1}\left(s_{O}^{j}\right) \\
\sigma_{p, q}^{j}\left(s_{R}^{j}\right)=-\gamma \rho_{p, q}^{j}\left(s_{R}^{j}\right)
\end{gathered}
$$

where

$$
s_{i}^{j}=\frac{r-r_{0}}{2 \sqrt{D_{i} t_{j}}} \quad(i=O, R)
$$




$$
\begin{gathered}
\xi_{i}^{j}=\frac{2 \sqrt{D_{i} t_{j}}}{r_{0}} \quad(i=O, R) \\
\chi^{j}=\left(k_{1}+k_{2}\right) t_{j}
\end{gathered}
$$

Thus, the concentration profiles (eqs. (A50)-(A52)) are totally determined. From the expressions for the concentration profiles, we can deduce those corresponding to the surface concentrations of species involved in a $\mathrm{CE}$ mechanism, which are given by eqs. (7)-(9) in theory. 